26 October, 2001

hep-ph/0110348

\title{
NUCLEAR PARTON DISTRIBUTIONS IN THE DGLAP APPROACH
}

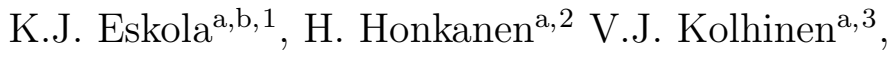 \\ P.V. Ruuskanen ${ }^{\mathrm{a}, \mathrm{b}, \text { 用 and C.A. Salgado }}$, 向 \\ a Department of Physics, University of Jyväskylä, \\ P.O.Box 35, FIN-40351 Jyväskylä, Finland \\ b Helsinki Institute of Physics, \\ P.O.Box 64, FIN-00014 University of Helsinki, Finland \\ c CERN, Theory Division, CH-1211 Geneva, Switzerland
}

\begin{abstract}
Determination of the nuclear parton distributions within the framework of perturbative QCD, the DGLAP equations in particular, is discussed. Scale and flavour dependent nuclear effects in the parton distributions are compared with the scale and flavour independent parametrizations of HIJING and of the Hard Probe Collaboration. A comparison with the data from deep inelastic lepton-nucleus scattering and the DrellYan process in proton-nucleus collisions is shown.
\end{abstract}

\footnotetext{
${ }^{1}$ kari.eskola@phys.jyu.fi

${ }^{2}$ heli.honkanen@phys.jyu.fi

${ }^{3}$ vesa.kolhinen@phys.jyu.fi

${ }^{4}$ vesa.ruuskanen@phys.jyu.fi

${ }^{5}$ carlos.salgado@cern.ch
} 


\section{Introduction}

In a high-energy collision of hadrons or nuclei $A$ and $B$, inclusive cross sections for the production of a particle $c$ involving a large scale $Q \gg \Lambda_{\mathrm{QCD}}$, can in the leading twist approximation be computed by using collinear factorization,

$$
\begin{gathered}
d \sigma\left(Q^{2}, \sqrt{s}\right)_{A B \rightarrow c+X}=\sum_{i, j=q, \bar{q}, g}\left[Z_{A} f_{i}^{p / A}\left(x_{1}, Q^{2}\right)+\left(A-Z_{A}\right) f_{i}^{n / A}\left(x_{1}, Q^{2}\right)\right] \otimes \\
\otimes\left[Z_{B} f_{j}^{p / B}\left(x_{2}, Q^{2}\right)+\left(B-Z_{B}\right) f_{j}^{n / B}\left(x_{2}, Q^{2}\right)\right] \otimes d \hat{\sigma}\left(Q^{2}, x_{1}, x_{2}\right)_{i j \rightarrow c+x}
\end{gathered}
$$

where $d \hat{\sigma}\left(Q^{2}, x_{1}, x_{2}\right)_{i j \rightarrow c+x}$ is the perturbatively calculable differential cross section for the production of $c$ at the scale $Q, x_{1,2} \sim Q / \sqrt{s}$ are the fractional momenta of the colliding partons $i$ and $j$, and $f_{i}^{p(n) / A}$ is the distribution of parton flavour $i$ in a proton (neutron) of the nucleus $A$, and correspondingly $f_{j}^{p(n) / B}$ is that for the nucleus $B$. The number of protons in $A(B)$ is denoted by $Z_{A}\left(Z_{B}\right)$. In the leading twist approximation, on which we shall focus in the following, multiple scattering of the bound nucleons does occur but all collisions are independent of each other and only one-parton densities are needed. At this level all the possible higher twist terms, suppressed by $1 / Q^{2}$ but enhanced by the nuclear geometry (thickness of the nuclei), are neglected. As correlations between partons are not considered, the nuclear effects enter only via the nuclear parton distribution functions $(\mathrm{nPDF}) f_{i}^{p, n / A, B}$. The nPDF differ from the parton distributions of the free proton but obey the same DGLAP [1] evolution equations. The DGLAP evolution of the nPDF has been studied in e.g. [2]-[9]. For studies of next-to-leading twist factorization involving two-parton distributions, see [10, 11].

The cross sections of hard processes measured in deeply inelastic $l A$ collisions and in $p A$ collisions offer the experimental constraints necessary for pinning down the parton densities in nuclei. Once the nPDF are known, the reference cross sections for hard probes of dense matter in ultrarelativistic nucleus-nucleus collisions can be computed and used in the search of the signals of the QGP. Another motivation for the study of the $\mathrm{nPDF}$ is that the measurements of certain hard processes in nuclear collisions are useful also for extracting information of the parton distributions of the free proton, provided that the contribution of the nuclear effects can first be removed [12].

The purpose of this note is to discuss the constraints of the nuclear parton densities within the DGLAP framework, such as the ones in [7, 8, 9]. We shall compare the outcome of the studies [7, 8], the EKS98 parametrization of the nuclear effects, with scale and flavour independent parametrizations of HIJING [13 and the Hard Probe Collaboration [14], and especially, with the data. 


\subsection{DIS and nPDF}

The cleanest way of getting information of the nPDF is from deeply inelastic leptonnucleus scattering (DIS) experiments. The differential cross section for deeply inelastic $l p$ scattering in the one-photon exchange approximation can be expressed as

$$
\frac{d \sigma^{l p}}{d Q^{2} d x}=\frac{4 \pi \alpha^{2}}{Q^{4}} \frac{F_{2}^{p}\left(x, Q^{2}\right)}{x}\left\{1-y-x y M / E_{l}+\frac{y^{2}}{2} \frac{1+4 x^{2} M^{2} / Q^{2}}{1+R\left(x, Q^{2}\right)}\right\},
$$

with the standard Lorentz-invariant variables $x=Q^{2} /(2 p \cdot q)$ and $y=p \cdot q / p \cdot k$, where $p, q$ and $k$ are the four-momentum of the proton, the exchanged virtual photon, and the incoming lepton, correspondingly. Virtuality of the photon is $Q^{2} \equiv-q^{2}$, mass of the nucleon is $M$, and $E_{l}$ is the energy of the incoming lepton in the target rest frame. In principle $0<x<A$ but for the discussion here the small cumulative tails of the distributions at $x>1$ can be safely neglected.

From the measurements with different nuclear targets, one knows that the ratio of the absorption cross sections of longitudinal and virtual photons with the target nucleons, $R\left(x, Q^{2}\right)=\sigma_{L}^{\gamma^{*}} / \sigma_{T}^{\gamma^{*}}$ does not significantly depend on the target nucleus $A$ at scales $Q^{2} \gtrsim 1.5 \mathrm{GeV}^{2}$ [15, 16]. The ratio of cross sections with different targets thus reflects the ratio of the nuclear structure functions $F_{2}^{A} / F_{2}^{B}$ :

$$
\frac{d \sigma^{l A} / d Q^{2} d x}{d \sigma^{l B} / d Q^{2} d x} \approx \frac{F_{2}^{A}\left(x, Q^{2}\right)}{F_{2}^{B}\left(x, Q^{2}\right)}=\frac{Z_{A} F_{2}^{p / A}\left(x, Q^{2}\right)+\left(A-Z_{A}\right) F_{2}^{n / A}\left(x, Q^{2}\right)}{Z_{B} F_{2}^{p / B}\left(x, Q^{2}\right)+\left(B-Z_{B}\right) F_{2}^{n / B}\left(x, Q^{2}\right)},
$$

where $F_{2}^{p, n / A, B}$ are the structure functions of bound nucleons. The ratio of the DIS cross sections from $l A$ and $l D$ is then related to the average structure functions per nucleon as

$R_{F_{2}}^{A}\left(x, Q^{2}\right) \equiv \frac{\frac{1}{A} d \sigma^{l A} / d Q^{2} d x}{\frac{1}{2} d \sigma^{l D} / d Q^{2} d x} \approx \frac{\frac{1}{A} F_{2}^{A}}{\frac{1}{2} F_{2}^{D}}=\frac{\frac{1}{2}\left(F_{2}^{p / A}+F_{2}^{n / A}\right)+\frac{1}{2}\left(\frac{2 Z}{A}-1\right)\left(F_{2}^{p / A}-F_{2}^{n / A}\right)}{\frac{1}{2}\left(F_{2}^{p / D}+F_{2}^{n / D}\right)}$,

where the numerator is written as a sum of isospin symmetric and non-symmetric terms. The nuclear effects in deuterium are small, less than 1 percent, so to a first approximation these can be neglected. Measurements of the ratio $R_{F_{2}}^{A}\left(x, Q^{2}\right)$ have revealed clear and systematic nuclear effects in different regions of Bjorken- $x$ [17]- 28]:

- "shadowing"; a depletion at $x \lesssim 0.1$,

- "anti-shadowing"; an excess at $0.1 \lesssim x \lesssim 0.3$,

- "EMC effect"; a depletion at $0.3 \lesssim x \lesssim 0.7$,

- "Fermi motion"; an excess towards $x \rightarrow 1$ and beyond. 
Systematics of $R_{F_{2}}^{A}$ in $A$ and in $x$ has been extensively studied e.g. in the experiments EMC [17, 18, 19], SLAC [20, 21], BCDMS [22], NMC [23]-[26], E665 [27, 28]. Since the $Q^{2}$ dependence of $R_{F_{2}}^{A}$ is quite weak at $x \gtrsim 0.1$ it has been more difficult to probe. Data with high enough precision, however, exist: the NMC has some years ago discovered a clear $Q^{2}$-dependence in the ratio of the cross sections $d \sigma^{\mu \mathrm{Sn}} / d \sigma^{\mu \mathrm{C}}$ [26], i.e. the scale dependence of the ratio $F_{2}^{\mathrm{Sn}} / F_{2}^{\mathrm{C}}$, at $x \sim 0.01$.

\section{The DGLAP analysis}

Generally, perturbative QCD (pQCD) cannot predict the absolute parton distributions. However, once the starting distributions are given at a scale $Q_{0}$, pQCD successfully predicts the evolution in $Q^{2}$ in the form of the DGLAP evolution equations [1]. The global DGLAP analyses of the parton distributions of the free proton, such as MRS [29] and CTEQ [30], involve a determination of those input distributions which, when evolved to different (higher) values of $Q$, give the best overall agreement with the data from different hard processes. Conservation of momentum and baryon number are maintained by the DGLAP evolution, and they are used as further constraints.

In the leading twist framework the situation is exactly the same for the nPDF: the data from hard processes in nuclear collisions at various values of $x$ and $Q$, together with the momentum and baryon number conservation, can be used to constrain the nonperturbative input distributions of partons in bound protons at some initial scale $Q_{0} \gg \Lambda_{\mathrm{QCD}}$. The link between the scales $Q_{0}$ and $Q$ is given by the DGLAP equations in the whole range of $x$ studied.

In general, the perturbative QCD scale evolution of the nPDF has been extensively studied in the literature, see e.g. [2]-[9] and [31, 32]. The origin of the nuclear effects is an interesting question but is beyond the scope of the DGLAP analysis. It has been suggested that for the DGLAP evolution one may compute the initial conditions at a scale $Q_{0}$ from a model, as is done e.g. in [3, 5], and then apply the DGLAP equations to describe the evolution in $Q$. Nevertheless, even in this case the key feature is the detailed comparison with the existing data. DGLAP analyses of the nPDF which attempt to rely only on the data in the determination of the initial conditions, are presented in Refs. [7, 8, 9].

\subsection{Quarks and antiquarks}

In the QCD-improved parton model (in leading order, or in the DIS-scheme in any higher order) the structure function $F_{2}$ of the proton(neutron) can be written in terms of its parton distributions as

$$
F_{2}^{p(n) / A}\left(x, Q^{2}\right)=\sum_{q=u, d, s, \ldots} e_{q}^{2}\left[x f_{q}^{p(n) / A}\left(x, Q^{2}\right)+x f_{\bar{q}}^{p(n) / A}\left(x, Q^{2}\right)\right] .
$$


As in the case of the free nucleons, the parton distributions of bound neutrons in isoscalar nuclei are obtained through isospin symmetry, $f_{u(\bar{u})}^{n / A}=f_{d(\bar{d})}^{p / A}$ and $f_{d(\bar{d})}^{n / A}=f_{u(\bar{u})}^{p / A}$. This is expected to be a good approximation for non-isoscalar nuclei as well.

It is convenient to define the nPDF for each parton flavor $i$ through the modifications of the corresponding distributions in the free proton,

$$
R_{i}^{A}\left(x, Q^{2}\right) \equiv \frac{f_{i}^{p / A}\left(x, Q^{2}\right)}{f_{i}^{p}\left(x, Q^{2}\right)},
$$

where we shall assume that the parton distributions of the free proton are fully known. For example, below the mass threshold of the charm quark, we can write

$$
R_{F_{2}}^{A}\left(x, Q^{2}\right)=\frac{5\left(u_{A}+\bar{u}_{A}+d_{A}+\bar{d}_{A}\right)+4 s_{A}+\left(\frac{2 Z}{A}-1\right) 3\left(u_{A}+\bar{u}_{A}-d_{A}-\bar{d}_{A}\right)}{5(u+\bar{u}+d+\bar{d})+4 s},
$$

where $u_{A} \equiv f_{u}^{p / A}=R_{u}^{A}\left(x, Q^{2}\right) f_{u}^{p}\left(x, Q^{2}\right)$, and similarly for the other quarks. The ratio $R_{F_{2}}^{A}$ measured at these scales thus constrains the individual ratios $R_{q}^{A}$ and $R_{\bar{q}}^{A}$ in certain combination.

Obviously, more constraints are needed in order to pin down the ratios $R_{i}^{A}$. These can be obtained from the measurements of the Drell-Yan dileptons in $p A$ collisions by E772 [33 and E866 [34] which offer high enough precision for getting statistically significant constraints. In the lowest order, the ratio of the differential cross sections for the Drell-Yan process in $p A$ and $p D$ collisions is given by

$$
\begin{aligned}
& R_{D Y}^{A}\left(x_{2}, Q^{2}\right) \equiv \frac{\frac{1}{A} d \sigma_{D Y}^{p A} / d x_{2} d Q^{2}}{\frac{1}{2} d \sigma_{D Y}^{p D} / d x_{2} d Q^{2}} \\
& =\left\{4\left[u_{1}\left(\bar{u}_{2}^{A}+\bar{d}_{2}^{A}\right)+\bar{u}_{1}\left(u_{2}^{A}+d_{2}^{A}\right)\right]+\left[d_{1}\left(\bar{d}_{2}^{A}+\bar{u}_{2}^{A}\right)+\bar{d}_{1}\left(d_{2}^{A}+u_{2}^{A}\right)\right]+4 s_{1} s_{2}^{A}+\ldots\right\} / N_{D Y} \\
& +\left(\frac{2 Z}{A}-1\right)\left\{4\left[u_{1}\left(\bar{u}_{2}^{A}-\bar{d}_{2}^{A}\right)+\bar{u}_{1}\left(u_{2}^{A}-d_{2}^{A}\right)\right]+\left[d_{1}\left(\bar{d}_{2}^{A}-\bar{u}_{2}^{A}\right)+\bar{d}_{1}\left(d_{2}^{A}-u_{2}^{A}\right)\right]\right\} / N_{D Y}
\end{aligned}
$$

where the invariant mass of the lepton pair is $Q^{2}$, and the subscript 2 (1) refers to the fractional momentum $x_{2}\left(x_{1}\right)$ of the parton from the target (proton). The denominator is

$$
N_{D Y}=4\left[u_{1}\left(\bar{u}_{2}+\bar{d}_{2}\right)+\bar{u}_{1}\left(u_{2}+d_{2}\right)\right]+\left[d_{1}\left(\bar{d}_{2}+\bar{u}_{2}\right)+\bar{d}_{1}\left(d_{2}+u_{2}\right)\right]+4 s_{1} s_{2}+\ldots
$$

with the dots denoting the heavier flavours. Again, the ratios $R_{q}^{A}$ and $R_{\bar{q}}^{A}$ are probed in certain combination. The typical $x$ and $Q^{2}$ range probed by the measurements of the ratios $R_{F_{2}}^{A}$ and $R_{D Y}^{A}$ can be seen in Fig. 1 .

In addition, conservation of baryon number,

$$
3=\int_{0}^{A} d x \sum_{q=u, d}\left[f_{q}^{p / A}\left(x, Q^{2}\right)-f_{\bar{q}}^{p / A}\left(x, Q^{2}\right)\right]
$$




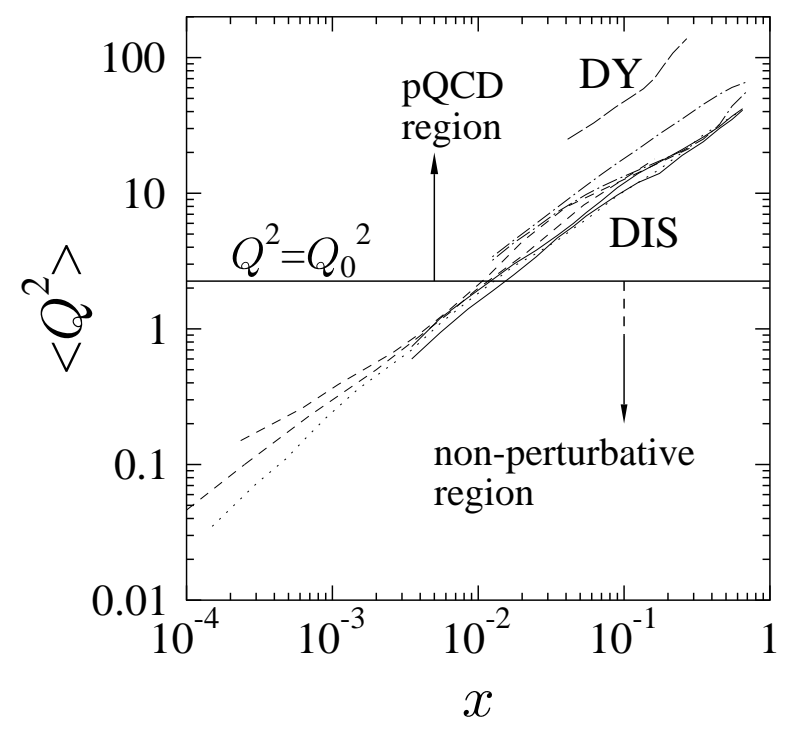

Figure 1: The correlation between the Bjorken $x$ and the photon virtuality $Q^{2}$ in deep inelastic $\mu A$ scattering measured by the NMC [23, 24, 25] and E665 [27, 28] (denoted by DIS). The same (with $x=x_{2}$ ) for the measurements of the Drell-Yan process in $p A$ by E772 [33] (denoted by DY). The starting scale of the DGLAP analysis [7] is $Q_{0}^{2}$.

$$
\approx \int_{0}^{1} d x \sum_{q=u, d}\left[R_{q}^{A}\left(x, Q^{2}\right) f_{q}^{p}\left(x, Q^{2}\right)-R_{\bar{q}}^{A}\left(x, Q^{2}\right) f_{\bar{q}}^{p}\left(x, Q^{2}\right)\right]
$$

can be used to pin down the valence quark distributions [3, 4. On the r.h.s. of Eq. (10), the small cumulative tails at $x>1$ have been neglected.

Even in an ideal case, where the experimentally measured DIS and DY ratios of Eqs. (4) and (8) would lie along a constant scale $Q_{0}$ in a wide range of $x$, the number of experimental constraints above would not be enough to fully fix the ratios $R_{q}^{A}\left(x, Q_{0}^{2}\right)$ and $R_{\bar{q}}\left(x, Q^{2}\right)$. In reality, the situations is even more difficult: the data on both DIS and DY are given only in distinct regions, within which the values of $x$ are strongly correlated with $Q^{2}$, as illustrated in Fig. 11. Therefore, a recursive procedure similar to that in the global analyses of the parton distributions of the free proton has to be adopted in order to determine the initial ratios $R_{i}^{A}\left(x, Q_{0}^{2}\right)$. Notice that in comparison with the free proton case, an additional variable, the mass number $A$, appears.

To simplify the determination of the input nuclear effects for valence and sea quarks (without invoking any specific model) one may in a leading approximation assume them to be separately flavor-independent: $R_{u_{V}}^{A}\left(x, Q_{0}^{2}\right) \approx R_{d_{V}}^{A}\left(x, Q_{0}^{2}\right) \approx R_{V}^{A}\left(x, Q_{0}^{2}\right)$, and $R_{\bar{u}}^{A}\left(x, Q_{0}^{2}\right) \approx R_{\bar{d}}^{A}\left(x, Q_{0}^{2}\right) \approx R_{s}^{A}\left(x, Q_{0}^{2}\right) \approx R_{S}\left(x, Q_{0}^{2}\right)$ [4, [7]. Note that this approximation is needed only at $Q_{0}^{2}$ but the observation in [7] is that it remains good also in the evolution to higher $Q^{2}$. In this approximation the problem reduces to constraining the three independent input ratios, $R_{V}^{A}, R_{S}^{A}$ and $R_{G}^{A}$ at the initial scale $Q_{0}^{2}$. The details 
of an analysis using this approach can be found in [0], below we only summarize the available constraints in each region of $x$.

- At $x \gtrsim 0.3$ the valence quark distributions dominate the structure function $F_{2}^{A}$, and $R_{F_{2}}^{A} \approx R_{V}^{A}$. The DIS data for $R_{F_{2}}^{A}$ therefore only constrains the magnitude of the EMC effect and the Fermi-motion in $R_{V}^{A}$ but not in $R_{S}^{A}$ or in $R_{G}^{A}$. For the sea quarks, it is assumed in [7] that $R_{S}\left(x \gtrsim 0.3, Q_{0}^{2}\right) \approx R_{V}\left(x \gtrsim 0.3, Q_{0}^{2}\right)$ (cf. the discussion for gluons below). To what extent the Drell-Yan production measurable in nuclear collisions at the SPS could probe the EMC effect of the nuclear sea quarks, was recently studied in Ref. 35.

- At $0.04 \lesssim x \lesssim 0.3$ the DIS and DY data both constrain the ratios $R_{S}^{A}$ and $R_{V}^{A}$ but from different regions of $Q^{2}$, as shown in Fig. 11. In addition, $R_{V}^{A}$ is restricted by conservation of baryon number. An outcome of the DGLAP analyses [3, 4, 7] is that at the input scale $Q_{0}^{2} \sim 2 \mathrm{GeV}^{2}$ the sea quark content of a nucleus remains smaller than that of the free proton, i.e. that no antishadowing appears in $R_{S}^{A}\left(x, Q_{0}^{2}\right)$.

- At $x \lesssim 0.04$ the DIS data for the ratio $R_{F_{2}}^{A}$ extend down to $x \sim 5 \cdot 10^{-3}$ in the region $Q \gtrsim 1 \mathrm{GeV}$ relevant for the DGLAP analysis. In the analyses [4, 7, 9] the nuclear valence quarks have less shadowing than the sea quarks, which is mainly due to the conservation of baryon number. Also more strongly shadowed valence quarks have been suggested [3].

- The DIS data for $R_{F_{2}}^{A}$ at $x \lesssim 5 \cdot 10^{-3}$ only exist in the region $Q \lesssim 1 \mathrm{GeV}$ which can be considered nonperturbative and not treatable with the DGLAP equations (see Fig. 11). A saturation behaviour, flattening of $R_{F_{2}}$ in $x \rightarrow 0$, is observed along the experimentally probed values of $Q^{2}$ [24, 27]. Such a behaviour, a weak dependence of $R_{F_{2}}^{A}$ on $x$, can also be expected at $Q_{0}^{2}$, provided that the sign of the slope of the $Q^{2}$ dependence of $R_{F_{2}}^{A}$ in the nonperturbative region remains the same (positive) as what is measured at $x \sim 0.01$ in the perturbative region [26]. The DIS data in the non-perturbative region should thus give a lower bound for $R_{F_{2}}^{A}\left(x, Q_{0}^{2}\right)$ at small values of $x$. The sea quarks dominate over the valence quarks in this region, so practically only the ratio $R_{S}^{A}$ is constrained by the DIS data.

\subsection{Gluons}

For the gluons the situation is less straightforward since direct measurements of the gluon distributions in nuclei are difficult. In principle the measurements of $D$-mesons in $p A$ collisions at various cms-energies can be used for pinning down the nuclear gluon distributions [36, 37]. These measurements already exist but so far the error bars in the results of experiment E789 [38] are too large for getting a stringent constraint for $R_{G}^{A}$. 
The future measurements of $D$ production in $p A$ collisions by the NA60 experiment at SPS, by PHENIX at RHIC and hopefully also by ALICE or CMS at the LHC, will provide very important input for fixing the gluon distributions in nuclei [37]. Also direct photons in nuclear collisions can be used for this purpose, possibly also diffractive scattering in DIS [39]. Production of $J / \Psi$ in $p A$ always involves strong final state effects $(J / \Psi$ suppression in normal nuclear matter $)$, which makes the extraction of the initial state effects from the data very difficult [40].

In the DGLAP analysis, the best (but still indirect) constraint on the ratio $R_{G}^{A}\left(x, Q^{2}\right)$ is provided by the measurements by NMC [26] of the $Q^{2}$ dependence of the ratio $F_{2}^{\mathrm{Sn}} / F_{2}^{\mathrm{C}}$. The scale evolution of $F_{2}$ is coupled to the gluon distributions at small values of $x$, where gluons dominate, approximately as 41

$$
\partial F_{2}\left(x, Q^{2}\right) / \partial \log Q^{2} \approx \frac{10}{27} \frac{\alpha_{s}}{\pi} x g\left(2 x, Q^{2}\right)
$$

Based on Eq. (11), the $Q^{2}$ slope of $R_{F_{2}}^{A}$ at small values of $x$ becomes

$$
\frac{\partial R_{F_{2}}^{A}\left(x, Q^{2}\right)}{\partial \log Q^{2}} \approx \frac{10 \alpha_{s}}{27 \pi} \frac{x g\left(2 x, Q^{2}\right)}{F_{2}^{D}\left(x, Q^{2}\right)}\left\{R_{G}^{A}\left(2 x, Q^{2}\right)-R_{F_{2}}^{A}\left(x, Q^{2}\right)\right\},
$$

which suggests that the more deeply gluons are shadowed, the slower is the evolution of $R_{F_{2}}^{A}$. So far only the NMC data [26] of the $Q^{2}$ dependence of the ratio $F_{2}^{\mathrm{Sn}} / F_{2}^{\mathrm{C}}$ has sufficient precision for getting stringent constraints, as first pointed out in Ref. [42].

Yet another indirect constraint, the momentum sum rule,

$$
1=\int_{0}^{A} d x \sum_{i=g, u, \bar{u}, \ldots} x f_{i}^{p / A}\left(x, Q^{2}\right) \approx \int_{0}^{1} d x \sum_{i=g, u, \bar{u}, \ldots} R_{i}^{A}\left(x, Q^{2}\right) x f_{i}^{p}\left(x, Q^{2}\right)
$$

should be taken into account in the DGLAP approach. A few percent flow of momentum from quarks and antiquarks to gluons is expected relative to the free proton case [3, 4. Since $\int_{0}^{1} d x R_{G}^{A}\left(x, Q_{0}^{2}\right) x g_{p}\left(x, Q_{0}^{2}\right)>\int_{0}^{1} d x x g_{p}\left(x, Q_{0}^{2}\right)$, antishadowing $R_{G}^{A}\left(x, Q_{0}^{2}\right)>1$ is bound to exist in some region of $x$. Below we summarize the constraints on $R_{G}^{A}\left(x, Q_{0}^{2}\right)$ in different regions of $x$.

- At $0.02 \lesssim x \lesssim 0.2$ the NMC data on the $Q^{2}$ evolution of the ratio $F_{2}^{\mathrm{Sn}} / F_{2}^{\mathrm{C}}[26]$ set the main constraint through the DGLAP evolution (the full equations; Eq. (12) holds at the small values of $x$ only). These NMC data extend down to $x=0.0125$, so only gluons at $x \gtrsim 0.02$ can be constrained. Especially, it is observed that the $Q^{2}$ slope of the ratio $F_{2}^{\mathrm{Sn}} / F_{2}^{\mathrm{C}}$ is clearly positive at small values of $x$, indicating that obviously also the $Q^{2}$ slope of $R_{F_{2}}^{A}\left(x, Q^{2}\right)$ is positive. Then, according to Eq. (12), $R_{G}^{A}\left(2 x, Q^{2}\right)>R_{F_{2}}^{A}\left(x, Q^{2}\right)$. The data thus seems to rule out the case where the shadowing of nuclear gluons is much stronger than the shadowing observed in $R_{F_{2}}^{A}$. In a solution consistent with the data on $F_{2}^{\mathrm{Sn}} / F_{2}^{\mathrm{C}}$ and with the momentum sum rule the gluons have less shadowing than the sea quarks and more antishadowing than the valence quarks or in $R_{F_{2}}^{A}$ [7, 42]. 
- At $x \lesssim 0.02$ stringent experimental constraints do not exist for the gluons at the moment. Assuming, however, that the $Q^{2}$ slope of $R_{F_{2}}^{A}$ remains positive, the measured saturation of shadowing in $R_{F_{2}}^{A}$ at $Q^{2} \ll 1 \mathrm{GeV}^{2}$ [24, 27] gives the lower bound for the shadowing of $R_{F_{2}}^{A}\left(x, Q_{0}^{2}\right)$. The weak $x$ dependence of $R_{F_{2}}^{A}$ (together with $\partial R_{F_{2}}^{A} / \partial Q^{2}>0$ ) indicates also a weak $x$ dependence of $R_{G}^{A}\left(x, Q_{0}^{2}\right)$, and it is then concievable to expect that $R_{G}^{A}\left(x, Q_{0}^{2}\right) \approx R_{F_{2}}^{A}\left(x, Q_{0}^{2}\right)$ for $x \ll 1$ [7]. The evolution is stable in the sense that the approximate equality remains true within about $5 \%$ even after the DGLAP evolution from $Q_{0} \sim 1 \mathrm{GeV}$ to $Q \sim 100$ $\mathrm{GeV}$ [8]. In order to pin down the gluons in this region, high-precision DIS measurements at small values of $x$ (but $Q^{2} \gtrsim 1 \mathrm{GeV}^{2}$ ) would be needed. More constraints in this region of $x$ are expected from the measurements of dileptons originating from the decays of the $D$ mesons in PHENIX at RHIC [36, 37] and in ALICE or CMS at the LHC [37].

- At $x \gtrsim 0.2$ there are currently no clear experimental constraints available for the gluons. Conservation of momentum does not reveal whether an EMC effect exists for the gluons or not: about $30 \%$ of the gluon momentum comes from $x \gtrsim 0.2$, so, say, a $10 \%$ net change in the momentum content of the EMC region can be compensated by roughly a $6 \%$ net effect in the region where the antishadowing bump is anticipated and which contains about half of the gluon momentum. Thus, the amount of antishadowing is not affected to the extent that it would violate the constraints obtained from $x \lesssim 0.2$ (see the estimates of the uncertainties in [42]). In the DGLAP evolution equations, the valence quarks act as source of gluons in the gluon evolution, and the gluons in turn feed the sea quark evolution. In course of the scale evolution, the experimentally verified EMC effect of valence quarks will generate a similar EMC effect for the gluons which in turn transmit the effect into the sea quark distributions, as seen in [4], where no input EMC effect was assumed for the gluons. A consistent assumption in the DGLAP framework therefore is to include an EMC effect already for the initial ratios $R_{G}^{A}$ and $R_{S}^{A}$. In this way the nuclear modifications $R_{i}^{A}$ remain stable against the evolution, i.e. they do not rapidly evolve away from their input values. In the future, however, experimental constraints for the nuclear gluons in this region of $x$ could be obtained from the measurements of dileptons (originating from the decays of the $D$ mesons) by the NA60 experiment at the SPS [37].

\subsection{The EKS98 parametrization}

We have discussed above how to get constraints for the nPDF. In the DGLAP framework [7] the problem boils down to determining the input distributions, i.e. the input ratios $R_{G}^{A}\left(x, Q_{0}^{2}\right), R_{V}^{A}\left(x, Q_{0}^{2}\right)$ and $R_{S}^{A}\left(x, Q_{0}^{2}\right)$. In practise, the extraction of the input ratios involves a recursive procedure: first the DGLAP evolution is performed with 
some input distributions, then a comparison with the data is made at various values of $x$ and $Q^{2}$, after which the input distributions are changed in order to achieve a better agreement with the data. This iterative procedure is repeated until the best set of $R_{G}^{A}\left(x, Q_{0}^{2}\right) R_{V}^{A}\left(x, Q_{0}^{2}\right)$ and $R_{S}^{A}\left(x, Q_{0}^{2}\right)$ is found. The details can be found in [7].

It is clear that the constraints always restrict the absolute $\mathrm{nPDF} f_{i}^{p / A}$ (see e.g. Eqs. (四) and (8)). Constraints for the ratios $R_{i}^{A}$ depend on the chosen set of parton distributions of the free proton, in terms of which the ratios in Eq. (6) are defined. Ideally of course there exists only one best set $\left\{f_{i}^{p}\right\}$, but in practise, as some uncertainties still appear also in the free proton level, several sets are in use, (MRS, CTEQ, GRV, etc.). To test how strongly the obtained nuclear effects might depend on the choice of the parton distributions of the free proton, we have repeated the analysis of [7] in [8] by using the CTEQ4L 44 distributions instead of the GRVLO distributions 43. In spite of the quite large differences in the gluon content (at small $x$ there are fewer gluons in CTEQ4L) and in the sea quarks (more flavour asymmetry in CTEQ4L) between these sets, the differences between $R_{i, \mathrm{GRV}}^{A}$ and $R_{i, \mathrm{CTEQ}}^{A}$ remained within a few percents only. Accepting this range of uncertainty, it is meaningful to prepare a "universal" parametrization for the ratios $R_{i}^{A}\left(x, Q^{2}\right)$. This task was performed in [8], and the parametrization, called "EKS98", is available for public use in the www 45] and now also in the latest version of the CERN PDFLIB [46].

\section{Comparison of different parametrizations}

Next we shall compare the EKS98-parametrization with two other parametrizations of the nuclear effects used in the literature, the default one in HIJING [13 and the one prepared in one of the Hard Probe Workshops [14]. Especially, we shall focus on the comparison of all these against the data.

\subsection{The HIJING parametrization}

The HIJING parametrization of the nuclear modifications of the parton distributions given in Ref. 13 is the following:

$R_{F_{2}}^{A}(x)=1+1.19(\ln A)^{1 / 6}\left[x^{3}-1.5\left(x_{0}+x_{L}\right) x^{2}+3 x_{0} x_{L} x\right]-\left[\alpha_{A}-\frac{1.08\left(A^{1 / 3}-1\right)}{\ln (A+1)} \sqrt{x}\right] \mathrm{e}^{-x^{2} / x_{0}^{2}}$

where $\alpha_{A}=0.1\left(A^{1 / 3}-1\right), x_{0}=0.1$ and $x_{L}=0.7$. The parametrization is based on fits to the old EMC data on $R_{F_{2}}^{A}[18$. In particular, in the applications of this parametrization, it is assumed that the modifications are identical for all parton flavours, and that the $Q^{2}$ dependence of the ratios is a negligible effect, $R_{i}^{A}\left(x, Q^{2}\right)=R_{F_{2}}^{A}(x)$. 


\subsection{The HPC parametrization}

An outcome of the Hard Probe Collaboration (HPC) meeting in ECT* at Trento (1995), was a parametrization [14] of $R_{F_{2}}^{A}(x)$. Similarly to the HIJING parametrization above, this parametrization assumes that the $Q^{2}$ dependence is a negligible effect. Possible differences between the modifications of different parton species are not considered, either. The HPC fit was motivated by a corresponding parametrization in [47] and it was obtained by fitting the re-analysed SLAC data [21] and re-analyzed NMC data [23]. The functional form of the HPC fit is

$$
R_{F_{2}}^{A}(x)= \begin{cases}R_{\mathrm{sh}} \frac{1+c_{D} c_{A}\left(1 / x-1 / x_{\mathrm{sh}}\right)}{1+c_{A} A^{p_{A}}\left(1 / x-1 / x_{\mathrm{sh}}\right)}, & x \leq x_{\mathrm{sh}} \\ a_{\mathrm{emc}}-b_{\mathrm{emc}} x, & x_{\mathrm{sh}} \leq x \leq x_{\mathrm{f}} \\ R_{f}\left(\frac{1-x_{f}}{1-x}\right)^{p_{\mathrm{f}}}, & x_{\mathrm{f}} \leq x \leq 1\end{cases}
$$

where the different regions are matched together by setting $R_{\mathrm{sh}}=a_{\mathrm{emc}}-b_{\mathrm{emc}} x_{\mathrm{sh}}$ and $R_{f}=a_{\mathrm{emc}}-b_{\mathrm{emc}} x_{f}$. In the EMC region $a_{\mathrm{emc}}=1+b_{\mathrm{emc}} x_{\mathrm{emc}}$. The $A$ dependence of $b_{\mathrm{emc}}$ is $b_{\mathrm{emc}}=p_{\mathrm{emc}}\left[1-A^{-1 / 3}-1.145 A^{-2 / 3}+0.93 A^{-1}+0.88 A^{-4 / 3}-0.59 A^{-5 / 3}\right]$ from Ref. 48. It was assumed (based on the data and the assumed $Q^{2}$ independence) that $R_{F_{2}}^{A}\left(x_{\mathrm{emc}}\right)=1$ and that the location of the EMC minimum is at $x_{f}$ independently of $A$. The eight fit parameters obtain the following values: $p_{A}=0.10011, c_{A}=0.0127343$, $c_{D}=1.05570 x_{\mathrm{sh}}=0.154037, x_{\mathrm{emc}}=0.275097, p_{\mathrm{emc}}=0.525080, x_{\mathrm{f}}=0.742059$ and $p_{\mathrm{f}}=0.320992$.

\subsection{The Comparison}

The results of the DGLAP analysis [0] for the ratios $R_{G}^{A}\left(x, Q^{2}\right), R_{S}^{A}\left(x, Q^{2}\right), R_{V}^{A}\left(x, Q^{2}\right)$, and $R_{F_{2}}^{A}$ at scales $Q^{2}=2.25 \mathrm{GeV}^{2}$ (thin solid lines), $14.7 \mathrm{GeV}^{2}$ (dashed), $108 \mathrm{GeV}^{2}$ (dotted-dashed) and $10000 \mathrm{GeV}^{2}$ (dashed) are compared with the HIJING parametrization (thick solid) and with the HPC parametrization (thick dashed) in Fig. 2. This figure is to illustrate two points: first, the $Q^{2}$ dependence becomes a non-negligible effect at small values of $x$. Secondly, the available experimental and sum-rule constraints lead to mutually quite different modifications for the valence quarks, sea quarks and gluons. For $A=208$, the HIJING parametrization underestimates the baryon number sum rule by $18 \%(24 \%)$ and the momentum sum rule by $13 \%(23 \%)$ at $Q^{2}=Q_{0}^{2}=2.25 \mathrm{GeV}^{2}$ $\left(Q^{2}=10^{4} \mathrm{GeV}^{2}\right)$. For the HPC parametrization the corresponding figures are $5 \%$ $(12 \%)$ for the baryon number and $8 \%$ (12\%) for the momentum sum rule. The effects of choosing different (lowest order) free proton parton distributions remain within one unit of percent. The deficit can easily be understood from the figure by comparing 


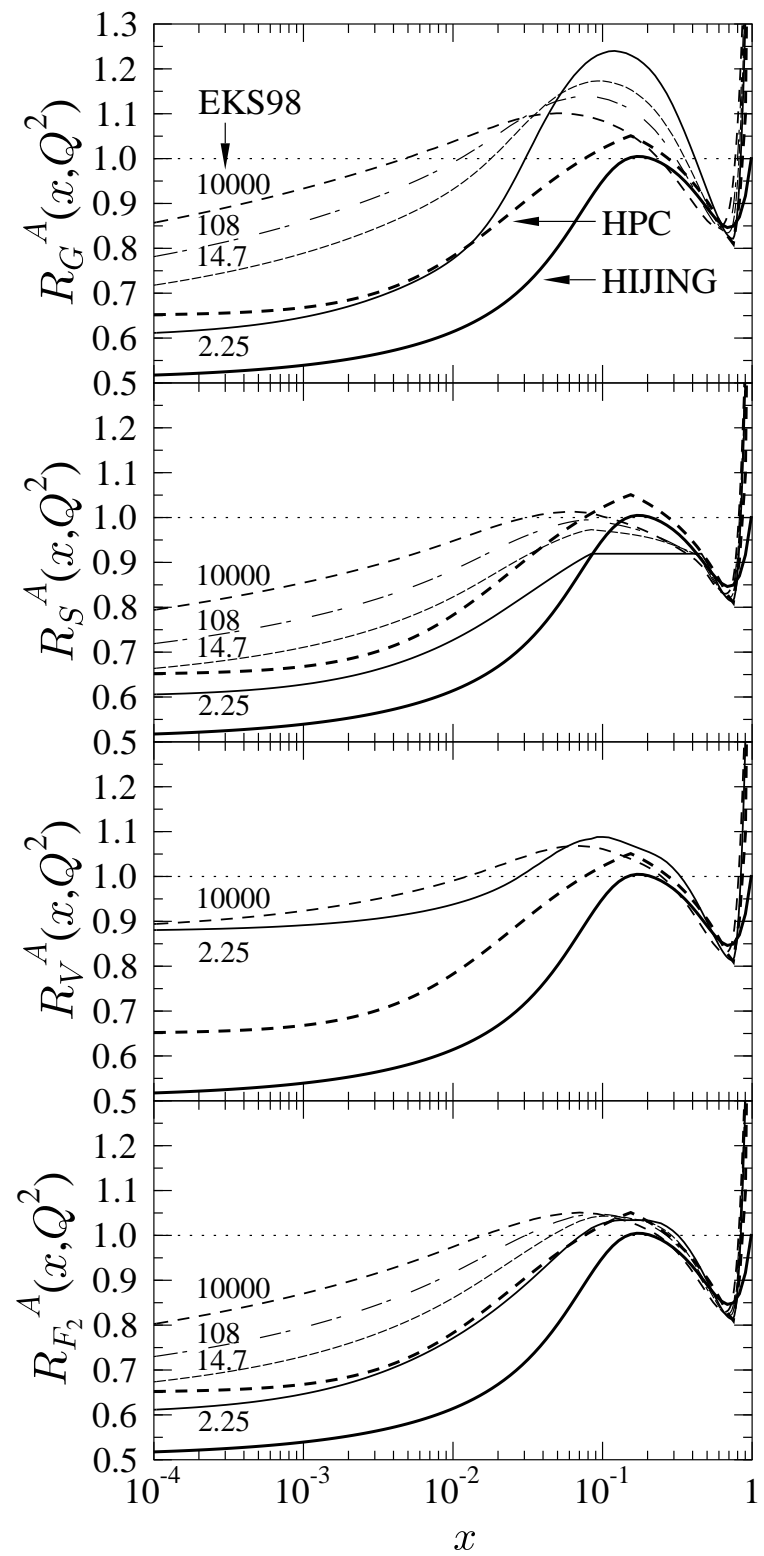

Figure 2. The nuclear modifications of parton densities for a heavy nucleus $A=$ 208 (isoscalar). The ratios $R_{G}^{A}\left(x, Q^{2}\right)$, $R_{S}^{A}\left(x, Q^{2}\right), \quad R_{V}^{A}\left(x, Q^{2}\right)$, and $R_{F_{2}}^{A}\left(x, Q^{2}\right)$ from Ref. [7] are denoted by EKS98, (thin lines, plotted at the fixed values of $Q^{2} / \mathrm{GeV}^{2}$ indicated on the left). The $Q^{2}$ independent parametrizations of the ratio $R_{F_{2}}^{A}(x)$ of HIJING (thick solid line) and that of HPC (thick dashed line) remain unchanged from panel to panel. The notation is the same in all panels.

with the EKS98 in which these conservation laws are met within an accuracy of $\sim 1 \%$ at all $Q^{2}$ for any (lowest order) PDF set of the free proton.

Fig. 3 shows the comparison of the EKS98 (open squares), HIJING (open diamonds) and HPC (open triangles) parametrizations with the NMC data [23] for $R_{F_{2}}^{A}$ (filled circles). The EKS98 results are computed by using CTEQ5L set of parton distributions [30, 46] and at scales $Q^{2}$ corresponding to the $\left\langle Q^{2}\right\rangle$ measured for each $x$ (see Fig. 1). The differences remain small between the EKS98, HPC and the data. HIJING agrees with the data for carbon but overestimates the $A$-dependence of shadowing for other nuclei. The error bars in the data represent the statistical and systematic errors added 


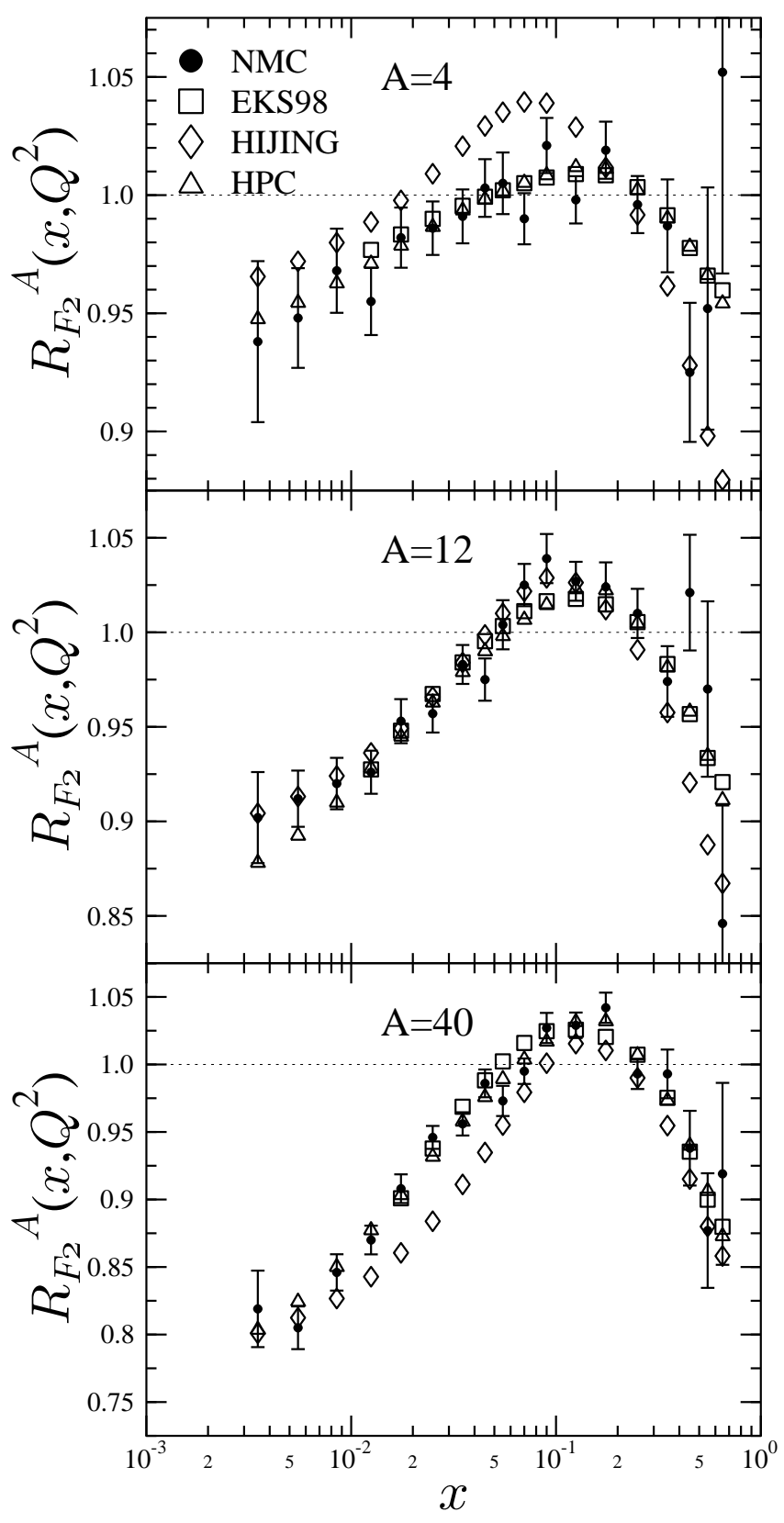

Figure 3. Comparison of the EKS98 (open squares), HIJING (open diamonds) and HPC (open triangles) parametrizations with the NMC data (filled circles with error bars) for $F_{2}^{A} / F_{2}^{D}$ for helium, carbon and calcium 23] . For EKS98 the comparison is only made for the experimental points above $Q^{2}=2.25 \mathrm{GeV}^{2}$. Notice that the vertical scales are different in each panel.

in quadrature.

Next, in Fig. 4 we show the comparison of EKS98, HIJING and HPC with the $A$ systematics of $R_{F_{2}}^{A}$ measured by the NMC [25]. Again, the data is shown by the filled circles with statistical errors (the inner error bars) and with the statistical and systematic errors added in quadrature (the outer error bars). The notation is the same as in the previous figure, and the EKS98 results are again computed at the scales 


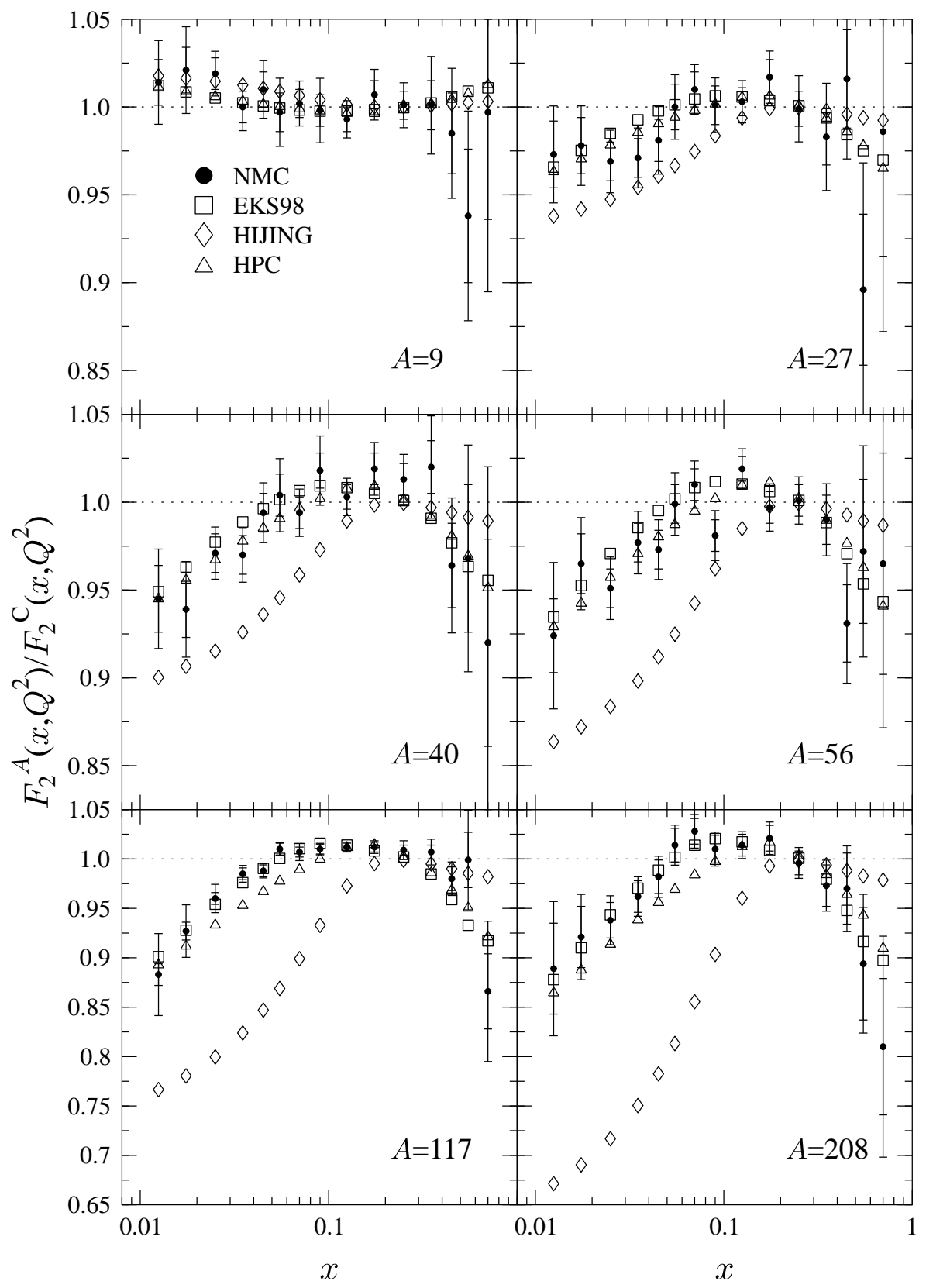

Figure 4. Comparison of the EKS98 (open squares), HIJING (open diamonds) and HPC (open triangles) parametrizations with the $\mathrm{NMC}$ data (filled circles with error bars) for $F_{2}^{A} / F_{2}^{\mathrm{C}}$ 25].

$Q^{2}=\left\langle Q^{2}\right\rangle$ for each value of $x$ given by the experiment. This figure shows that the $A$ dependence of shadowing is clearly too strong in HIJING. It should also be noted that the data of Fig. 4 was not yet available for the HPC fit but that the HPC parametrization falls nevertheless fairly close to the data. Notice that of the data sets shown in Fig. 4, the one for $\mathrm{Sn} / \mathrm{C}$ gives the most stringent constraint for any fit, due 
to the smallest error bars.

In Fig. 5, we have plotted the $Q^{2}$ dependence of the ratio $F_{2}^{\mathrm{Sn}} / F_{2}^{\mathrm{C}}$ at fixed values of $x$ corresponding to those in the NMC data [26]. The EKS98 (with CTEQ5L distributions) is shown by the solid lines, HIJING by the dotted lines and HPC with the dashed lines. The data is shown by the open squares with (statistical) error bars. At small values of $x$ the $Q^{2}$ dependence is not a negligible effect.

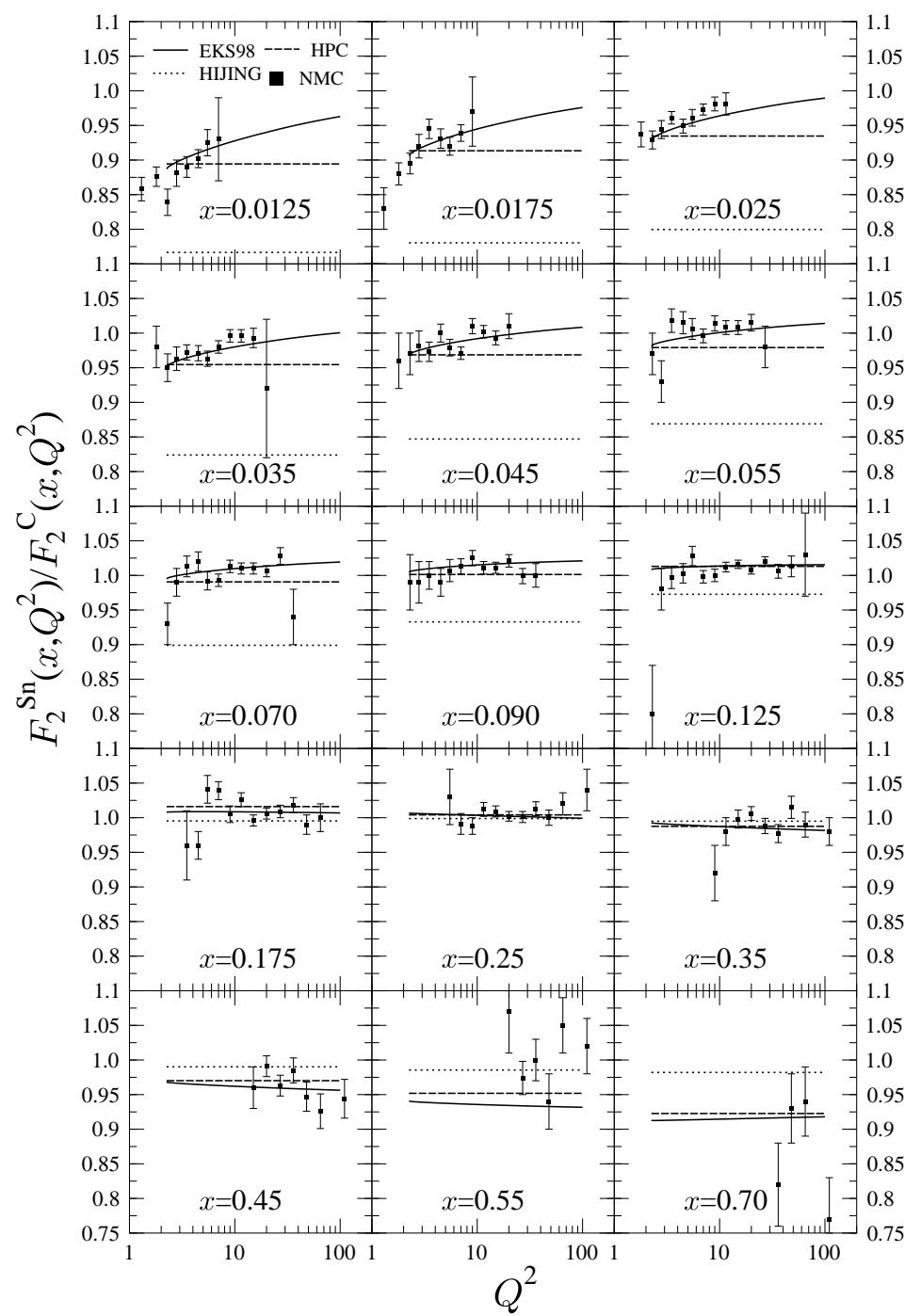

Figure 5. The $Q^{2}$ dependence of the ratio $F_{2}^{\mathrm{Sn}} / F_{2}^{\mathrm{C}}$ for fixed values of $x$. The EKS98 results are the solid lines, HIJING parametrization the dotted line and the HPC parametrizaton the dashed line. The NMC data [26] is shown by the filled circles with (statistical) error bars.

Finally, in Fig. 6 we show the comparison of EKS98, HIJING and HPC with the E772 DY data [33] in $p A$ collisions. In the computation of the ratio from Eq. (8), 


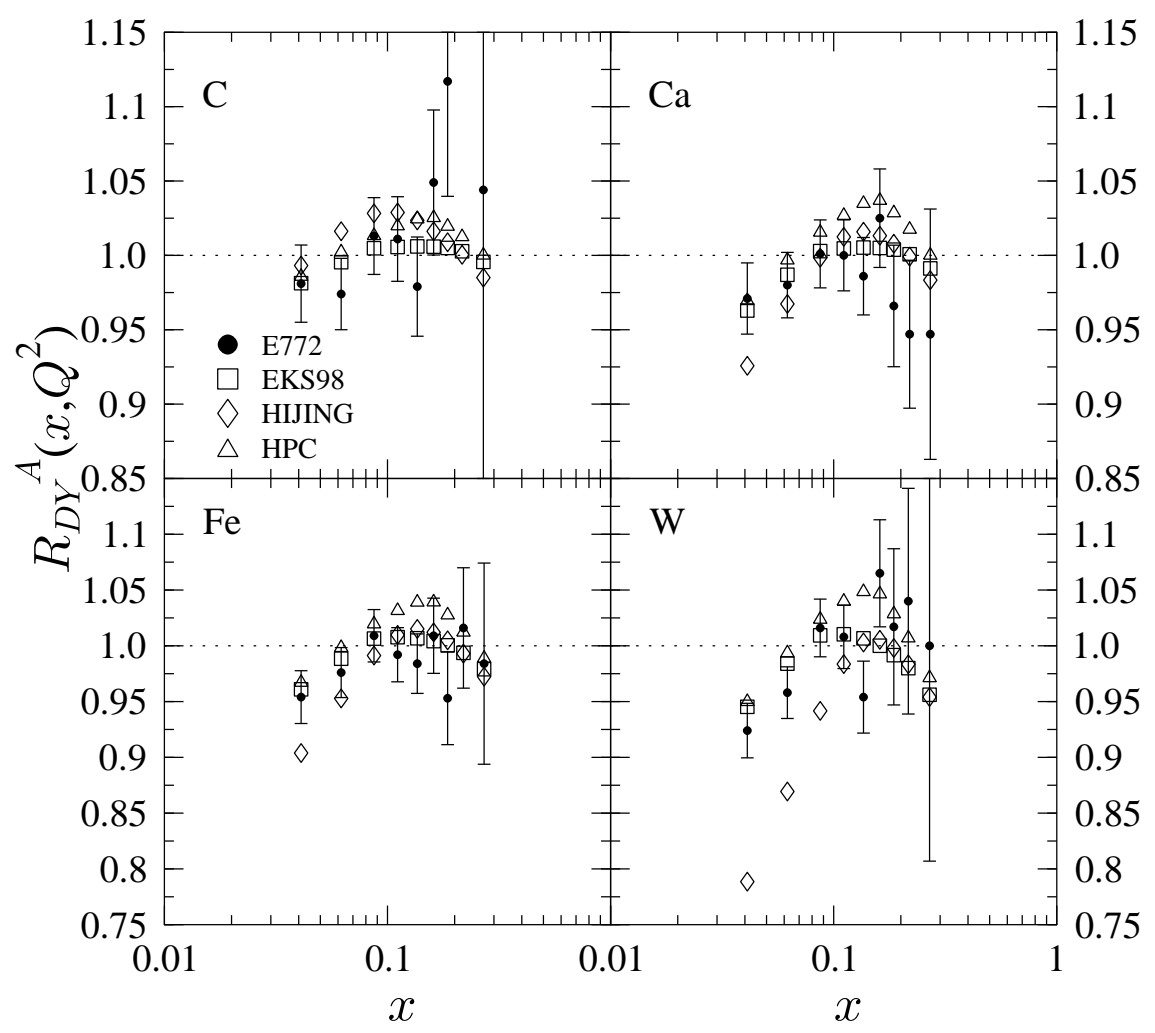

Figure 6. The Drell-Yan ratio $R_{D Y}^{A}\left(x_{2},\left\langle Q^{2}\right\rangle\right)$ computed from Eq. (8). The E772 data [33] are shown by the filled circles with error bars, the EKS98 results by the open squares, the HIJING parametrization by the open diamonds, and the HPC parametrization by open triangles.

the CTEQ5L distributions [30, 46] of the free proton have been used. The scales $Q^{2}$ for each $x_{2}$ used in computing the EKS98 results are those in Fig. 1. Also here the conclusion is that the HIJING parametrization clearly overestimates the $A$ dependence of shadowing.

\section{Conclusions}

In this note we have discussed the determination of the nuclear parton distributions in the lowest order leading twist DGLAP framework. We have shown which kinematical range and which combinations of the parton distributions are probed in the measurements of the deeply inelastic $l A$ scatterings and the Drell-Yan process in $p A$ collisions, and to what extent the measurements offer constraints for the input distributions. The remaining uncertainties are also discussed.

To demonstrate the differences between the different parametrizations of the nuclear effects used in the literature, we have compared the EKS98, HIJING and HPC 
parametrizations with each other and, most importantly, with the data. The HIJING parametrization for $R_{F_{2}}^{A}$ clearly overestimates the $A$ dependence of nuclear shadowing, and leads to a contradiction with the data. Our conclusion therefore is that for detailed studies of shadowing effects the HIJING parametrization [13] is not an adequate description of the nuclear modifications of quark and antiquark distributions. If the flavour and scale dependence of the nuclear effects could be neglected for some applications, we note that the HPC parametrization gives a better representation of the data for the ratios $R_{F_{2}}^{A}$ than the one in [13]. For such a use the HPC fit should, however, be redone by including the extensive $A$ systematics provided by the NMC measurements [25]: especially the data on the ratio $F_{2}^{\mathrm{Sn}} / F_{2}^{\mathrm{C}}$ offers an additional constraint for the fit.

In the leading twist framework also the conservation of baryon number and momentum should be required, since all the nuclear effects are contained in the parton distributions. If so done, the modifications for the valence quarks, sea quarks and gluons differ from each other, as is demonstrated in the DGLAP analyses [3, 4, 7, 8, 9]. If the HIJING or HPC parametrizations of $R_{F_{2}}^{A}(x)$ are directly used to modify the distribution of all parton flavors, the baryon number will be underestimated for $A=208$ by $18 \ldots 24 \%$ with HIJING and $5 \ldots 12 \%$ with HPC at scales $Q^{2}=2.25 \ldots 10^{4} \mathrm{GeV}^{2}$.

The NMC measurements 26 have revealed a clear $Q^{2}$ dependence in the ratio $F_{2}^{\mathrm{Sn}} / F_{2}^{\mathrm{C}}$, at $x \sim 0.01$. Naturally, scale independent parametrizations cannot reproduce the observed behaviour. In a DGLAP framework, the $Q^{2}$ dependence of the ratio $F_{2}^{\mathrm{Sn}} / F_{2}^{\mathrm{C}}$ can be used to constrain the nuclear gluon distributions, as first suggested in Ref. [42]. In the DGLAP analysis [7] it was shown that the constraints obtained for the gluons 42] are also consistent with the momentum sum rule, i.e. that a fairly strong antishadowing of gluons appears at the input scale $Q_{0}^{2} \sim 2 \mathrm{GeV}^{2}$. The HIJING parametrization underestimates the momentum sum for $A=208$ by $13 \ldots 23 \%$ and the HPC by $8 \ldots 12 \%$ at scales $Q^{2}=2.25 \ldots 10^{4} \mathrm{GeV}^{2}$. In addition, within the DGLAP analysis, the data [26] seems to rule out the case such as the Ansatz 2 in Ref. [4, where gluons would have clearly stronger shadowing than that observed in $R_{F_{2}}^{A}$.

Finally, the analysis of Ref. [7] which lead to the EKS98 parametrization [8] should be improved within the DGLAP framework in obvious ways: the fitting procedure should be automated for inclusion of new data sets in the future, the parameter space should be more thoroughly explored to estimate better the uncertainties (see also [9]), and next-to-leading order analysis should be performed. We do not, however, expect the results change very much from the EKS98 [7, 8]. One should also consider expansions of the DGLAP framework, such as an inclusion of the recombination terms [49, 50] in the evolution equations [2, 4], parton saturation phenomena at small values of $x$ [49][53], and possible higher-twist effects in the cross sections [10, 11], especially at lower scales. Also input from the different models for the origin of the nuclear effects can be considered. However, the comparison with the data should remain as the key feature of the analysis, since it is (at least presently) not possible to compute the absolute nuclear parton distributions from first principles. 


\section{Acknowledgements}

We thank the members of the Hard Probe Collaboration for several discussions on the subjects considered in this paper. The financial support from the Academy of Finland, grant no. 773101, is gratefully acknowledged. C.A.S. has been supported by a Marie Curie Fellowship of the European Community programme TMR (Training and Mobility of Researchers), under the contract number HPMF-CT-2000-01025.

\section{References}

[1] Yu. Dokshitzer, Sov. Phys. JETP 46 (1977) 1649; V.N. Gribov and L. N. Lipatov, Sov. Nucl. Phys. 15 (1972) 438, 675; G. Altarelli, G. Parisi, Nucl. Phys. B 126 (1977) 298.

[2] J. Qiu, Nucl. Phys. B 291 (1987) 746.

[3] L. L. Frankfurt, M. I. Strikman and S. Liuti, Phys. Rev. Lett. 65 (1990) 1725.

[4] K.J. Eskola, Nucl. Phys. B 400 (1993) 240.

[5] S. Kumano, Phys. Rev. C 50 (1994) 1247; S. Kumano and M. Miyama, Phys. Lett. B 378 (1996) 267.

[6] D. Indumathi and W. Zhu, Z. Phys. C 74 (1997) 119 hep-ph/9605417.

[7] K.J. Eskola, V.J. Kolhinen and P.V. Ruuskanen, Nucl. Phys. B 535 (1998) 351 hep-ph/9802350].

[8] K.J. Eskola, V.J. Kolhinen and C.A. Salgado, Eur. Phys. J. C 9 (1999) 61 hep$\mathrm{ph} / 9807297$

[9] M. Hirai, S. Kumano and M. Miyama, Phys. Rev. D 64 (2001) 034003 hep$\mathrm{ph} / 0103208$.

[10] J. Qiu and X. Zhang, hep-ph/0109210; X.-F. Guo, J. Qiu and W. Zhu, hepph/0110038.

[11] R. J. Fries, A. Schäfer, E. Stein and B. Müller, Nucl. Phys. B 582 (2000) 537 hep-ph/0002074; V. Braun, R. J. Fries, N. Mahnke and E. Stein, Nucl. Phys. B 589 (2000) 381 [Erratum-ibid. B 607 (2000) 433] hep-ph/0007279].

[12] K. Long, R. Nisius and W. J. Stirling, hep-ph/0109092.

[13] X. Wang and M. Gyulassy, Phys. Rev. D 44 (1991) 3501. 
[14] Jan Czyzewski, K.J. Eskola, and J. Qiu, at the III International Workshop on Hard Probes of Dense Matter, ECT*, Trento, June 1995.

[15] M. Arneodo, Phys. Rep. 240 (1994) 301.

[16] K. Ackerstaff et al. [HERMES Collaboration], Phys. Lett. B 475 (2000) 386 hepex/9910071].

[17] EMC collaboration, J.J. Aubert et al., Phys. Lett. B 123 (1983) 275; A. Bodek et al., Phys. Rev. Lett. 50 (1983) 1431; 51 (1983) 534.

[18] EMC collaboration, J. Ashman et al., Phys. Lett. B 202 (1988) 603; M. Arneodo et al., Phys. Lett. B 211 (1988) 493.

[19] M. Arneodo et al., Nucl. Phys. B 333 (1990) 1.

[20] R.G. Arnold et al., Phys. Rev. Lett. 52 (1984) 727.

[21] J. Gomez et al., Phys. Rev. D 49 (1994) 4348.

[22] BCDMS collaboration, G. Bari et al., Phys. Lett. B 163 (1985) 282.

[23] NMC collaboration, P. Amaudruz et al., Nucl. Phys. B 441 (1995) 3.

[24] NMC collaboration, M. Arneodo et al., Nucl. Phys. B 441 (1995) 12.

[25] NMC collaboration, M. Arneodo et al., Nucl. Phys. B 481 (1996) 3.

[26] NMC collaboration, M. Arneodo et al., Nucl. Phys. B 481 (1996) 23.

[27] E665 collaboration, M.R. Adams et al., Phys. Rev. Lett. 68 (1992) 3266.

[28] E665 collaboration, M.R. Adams et al., Z. Phys. C 67 (1995) 403.

[29] A.D. Martin, R.G. Roberts, W.J. Stirling and R.S. Thorne, Eur. Phys. J. C4 (1998) 463.

[30] H. L. Lai et al. [CTEQ Collaboration], Eur. Phys. J. C 12 (2000) 375 hep$\mathrm{ph} / 9903282$.

[31] Z. Huang, H. J. Lu and I. Sarcevic, Nucl. Phys. A 637 (1998) 79 hep-ph/9705250.

[32] A. L. Ayala, M. B. Gay Ducati and E. M. Levin, Nucl. Phys. B 493 (1997) 305 hep-ph/9604383.

[33] D. M. Alde et al., Phys. Rev. Lett. 64 (1990) 2479.

[34] E866 Collaboration, M.A. Vasiliev et al., Phys. Rev. Lett. 83 (1999) 2304. 
[35] K. J. Eskola, V. J. Kolhinen, C. A. Salgado and R. L. Thews, JYFL-4-00, hepph/0009251, Eur. Phys. J. C in press.

[36] Z. Lin and M. Gyulassy, Phys. Rev. Lett. 77 (1996) 1222 nucl-th/9510041]; Phys. Rev. C 51 (1995) 2177 [Erratum-ibid. C 52 (1995) 440] nucl-th/9409007.

[37] K. J. Eskola, V. J. Kolhinen and R. Vogt, JYFL-6-01, hep-ph/0104124, Nucl. Phys. A, in press.

[38] M.J. Leitch et al., Phys. Rev. Lett. 7219942542.

[39] L. Frankfurt and M. Strikman, Eur. Phys. J. A 5 (1999) 293 hep-ph/9812322.

[40] C. A. Salgado, hep-ph/0105231, Phys. Lett. B, in press.

[41] K. Prytz, Phys. Lett. B 311 (1993) 286.

[42] T. Gousset and H.J. Pirner, Phys. Lett. B 375 (1996) 349 hep-ph/9601242.

[43] M. Glück, E. Reya and A. Vogt, Z. Phys. C 53 (1992) 53.

[44] H.L. Lai et al., Phys. Rev. D 55 (1997) 1280.

[45] http://urhic.phys.jyu.fi/, http://www-fp.usc.es/phenom

[46] H. Plothow-Besch, Int. J. Mod. Phys. A 10 (1995) 2901; Comput. Phys. Comm. 75 (1993) 396; PDFLIB User's Manual - Version 8.04, W5051 PDFLIB, 2000.04.17, CERN-ETT/TT.

[47] C. J. Benesh, J. Qiu and J. P. Vary, Phys. Rev. C 50 (1994) 1015 hep-ph/9403265.

[48] G. I. Smirnov, Phys. Atom. Nucl. 58 (1995) 1613 [Yad. Fiz. 58 (1995) 1712] hepph/9502368.

[49] L.V. Gribov, E.M. Levin and M.G. Ryskin, Phys. Rep. 100 (1983) 1.

[50] A.H. Mueller and J. Qiu, Nucl. Phys. B 268 (1986) 427.

[51] L. D. McLerran and R. Venugopalan, Phys. Rev. D 49 (1994) 3352 hepph/9311205]; Phys. Rev. D 49 (1994) 2233 [hep-ph/9309289;; A. Ayala, J. JalilianMarian, L. D. McLerran and R. Venugopalan, Phys. Rev. D 53 (1996) 458 hepph/9508302].

[52] D. Kharzeev and E. Levin, nucl-th/0108006.

[53] N. Armesto and C. A. Salgado, Phys. Lett. B 520 (2001) 124 hep-ph/0011352. 